\title{
High speed determination of laser wavelength using Poincaré descriptors of speckle
}

\author{
Laura O'Donnell ${ }^{\mathrm{a}}$, Kishan Dholakia, ${ }^{\mathrm{a}, \mathrm{b}}$, Graham D. Bruce ${ }^{\mathrm{a}}$ \\ ${ }^{a}$ SUPA School of Physics and Astronomy, University of St Andrews, North Haugh, St Andrews, UK, KY16 $9 S S$ \\ ${ }^{b}$ Department of Physics, College of Science, Yonsei University, Seoul 03722, South Korea
}

\begin{abstract}
Laser speckle can provide a powerful tool that may be used for metrology, for example measurements of the incident laser wavelength with a resolution beyond that which may be achieved in a commercial device. However, to realise highest resolution requires advanced multi-variate analysis techniques, which limit the acquisition rate of such a wavemeter. Here we show an arithmetically simple method to measure wavelength changes with dynamic speckle, based on a Poincarè descriptor of the speckle pattern. We demonstrate the measurement of wavelength changes at femtometer-level with a measurement time reduced by two orders of magnitude compared to the previous state-of-the-art, which offers promise for applications such as speckle-based laser wavelength stabilisation.
\end{abstract}

Keywords: speckle, wavelength measurement, Poincaré descriptors

\section{Introduction}

Shaping of the spatial profile of light to a pre-determined pattern of interest has allowed major advances in diverse areas such as optical manipulation, imaging and communications 1. A commonly underappreciated method of beam shaping is to randomize the spatial profile of the light to create a speckle pattern. The randomized intensity pattern has been extensively used in the field of ultracold atoms as a controlled source of noise or disorder [2, 3, 4. Moreover, as the interference processes leading to the formation of speckle are linear, deterministic and reversible [5], dynamic changes in the speckle pattern can be exploited as an exquisite sensor for changes in the scattering medium [6, 7, 8 , or in the illuminating light field itself [9, 10, 11, 12, 13, 14, 15, 16, 17, 18, 19, 20, 21, 22, 23.

Here, we explore a new method to use dynamic speckle to probe changes of the wavelength of a laser beam. The formation of speckle can be thought of as a highly complex interferometer, where the speckle grains are the analog of interferometer fringes. These grains thus have a wavelength-dependent size and position and can therefore, after an appropriate training procedure, be used as a marker for wavelength. The transmission matrix method [18, 21] is capable of measuring these changes over a range as large as $400 \mathrm{~nm}$ to $1100 \mathrm{~nm}$ at a resolution down to the speckle correlation limit, which can be as low as $1 \mathrm{pm}$, while principal component analysis (PCA) 14, 17, 20 can be used to improve this resolution down to the attometer level. This high-resolution and broad range compares well with current high-end wavemeters, while offering a sim-

\footnotetext{
* Corresponding author

Email address: gdb2@st-andrews.ac.uk (Graham D. Bruce)
}

ple, compact and relatively inexpensive setup. This configuration also realises a speckle-based spectrometer with picometer-level resolution [18.

Among the application areas for such a high-accuracy wavemeter is the option of wavelength-stabilising a laser without the need for a spectroscopic reference [24]. Stabilising the wavelength of a laser using a speckle-based setup locks only to a speckle pattern of interest. Therefore, the lock-point is freely chosen. However, the maximum lock update rate previously achieved using PCA is $200 \mathrm{~Hz}$ [17. This is relatively slow compared to modern laser frequency controllers which are designed to provide current feedback to compensate $\mathrm{kHz}$-rate frequency noise. A dynamic speckle wavemeter which can measure wavelength changes on the ms-scale was reported in [25], but the wavelength resolution was restricted to the picometre regime, necessitating the search for a fast and precise speckle analysis technique.

A change in wavelength on a scale below the speckle correlation limit causes only grain-scale changes to the speckle pattern. Recently, a technique involving Poincaré descriptors was developed to investigate the size of speckle grains [26, 27]. Here we show that the grain-scale variations of speckle patterns that are induced by changes of the wavelength $\lambda$ are also captured by a Poincaré descriptor. Below the correlation limit, this metric varies monotonically with wavelength, making it a suitable tool for measuring wavelength in this regime. We demonstrate the measurement of femtometer-level wavelength changes using laser speckle generated from an integrating sphere. Finally, we show that the method offers a huge increase in computation speed relative to PCA, making it an interesting candidate for devices which require accurate, $\mathrm{kHz}$-rate measurements of wavelength. 


\section{Poincaré descriptor for wavelength measure- ment}

To carry out Poincaré analysis, each speckle pattern $I_{x, y}(\lambda)$, where $x, y$ denote spatial co-ordinates, is normalised with respect to its maximum value in order to remove intensity fluctuations between patterns. The Poincaré descriptor $P_{k}(\lambda)$ of an individual pattern is the standard deviation of the intensity difference between rows separated by a fixed distance $k$, i.e.

$$
P_{k}(\lambda)=\operatorname{std}\left(\sum_{y} I_{x, y}(\lambda)-\sum_{y} I_{x+k, y}(\lambda)\right) .
$$

This Poincare descriptor is otherwise known as the standard deviation of lag- $k$ differences, related to the standard deviation of successive differences 28, which is widely used in areas such as heart rate variability analysis [29, 30. It was recently demonstrated that this metric can probe short-range correlations in the spatial and temporal structure of speckle [26, 27. In order to track grain-scale changes of speckle patterns, we set the parameter $k$ to the size of a typical speckle in the image, determined using the methods outlined in 26 . The variation with wavelength must be determined empirically, via a training phase which extracts a scaling law between $P_{k}$ and $\lambda$. We note that the choice of whether to compare rows, columns or indeed any radial direction is arbitrary, provided the lag $k$ is set appropriately to the speckle grain size along that axis.

To demonstrate the method, we first simulate the speckle patterns produced for each of a range of wavelengths incident upon a multiply-scattering medium, using the model previously presented in 17]. The simulation assumes a linearly-polarised and monochromatic incident Gaussian beam of a particular power, wavelength and fixed spot size. To generate speckle, the optical field is propagated through a path of multiple equally-spaced random phase plates using paraxial wave theory and the split-step beam propagation method 31. Each phase retarder is a matrix of randomly-assigned, spatially slowlyvarying phase retardations, with a small refractive index difference to the air $(\Delta n=0.001)$ ensuring the prominent effect is to randomly scatter light in the forward direction. The number and separation of the retarders is set to mimic a $38.1 \mathrm{~mm}$-diameter, spectralon-coated integrating sphere, with the number given by the average number of reflections within the sphere (calculated from the sphere multiplier 32 to be 20) and the separation set to the sphere diameter. In practice, this simulation underestimates the randomization of the light field by the integrating sphere, as many wavelets in the sphere undergo significantly more reflections than the mean, making the speckle spectral correlation length much shorter. For this reason, we emphasize results in this section relative to the speckle spectral correlation length. The resulting intensity distributions are discretized to integers between 0 and 255 , and a spatial subset is sampled onto a $256 \times 256$ pixe- lated grid, to mimic the limited dynamic range and spatial extent of typical cameras.

Speckle patterns for wavelengths between $780 \mathrm{~nm}$ and $781 \mathrm{~nm}$ (in 4 pm steps) are generated for Poincaré analysis. The exact form of $P_{k}(\lambda)$ depends on the microscopic detail of the scattering structure, the image size and the value of $k$. Therefore, for a particular experimental apparatus to function as a wavemeter, $P_{k}(\lambda)$ must first be determined in a training phase. An example of the dependency of $P_{3}$ on wavelength is shown in Figure 1(a). In this example, $P_{k}$ varies monotonically with wavelength over a range $\sim$ $430 \mathrm{pm}$. Therefore, for wavelength changes smaller than this bandwidth, the Poincaré descriptor is a valid metric for extracting the wavelength. The bandwidth remains approximately constant for changes of image acquisition parameters and $k$, provided that $k$ is comparable to and above the speckle grain size (in our simulations, the spatial correlation function of the generated speckle falls to $1 / e$ at $\sim 2.8$ pixels). However, the upper and lower limits of the monotonic range can be tuned by judicious choice of $k$ and image acquisition parameters. This flexibility can be exploited during a training phase, in order to centre the range of operation and to ensure a non-zero derivative of $P_{3}(\lambda)$ around a wavelength of interest.

The finite bandwidth is related to the speckle correlation length. The speckle patterns produced by two distinct wavelengths are similar for small wavelength separations, which gives rise to the continuous variation of $P_{k}(\lambda)$. However, the speckle patterns are highly dissimilar for large wavelength separations. The scale over which the speckle decorrelates is known as the speckle correlation limit, and gives a lower-bound to the resolution of the transmission matrix method for wavelength characterisation [18. This is quantified as the wavelength separation for which the speckle patterns have a correlation coefficient of $50 \%$. Figure 1(b) shows the Pearson correlation coefficient between the speckle pattern produced with each wavelength in our test set, and the speckle pattern at $\lambda=780.000 \mathrm{~nm}$. The speckle correlation falls to $50 \%$ for a wavelength separation of $307 \mathrm{pm}$. This highlights the complimentary nature of the Poincaré descriptor method and the transmission matrix method: to extract the wavelength, the former requires that the speckles patterns have a sufficiently high correlation coefficient, while the latter relies on the speckle patterns produced by two wavelengths being uncorrelated. Thus the Poincaré descriptor method is a natural partner for use in tandem with the transmission matrix method, to increase the dynamic range over which wavelength can be measured.

\section{Experimental demonstration of wavelength re- covery using the Poincaré descriptor method}

We test the Poincaré method experimentally, using laser light from an external cavity diode laser (ECDL) (Laser diode: Toptica LD-0785-P220; Housing: Toptica DL-100) which is stabilised to the Rb-87 D2 line $(F=2 \rightarrow F=$ 

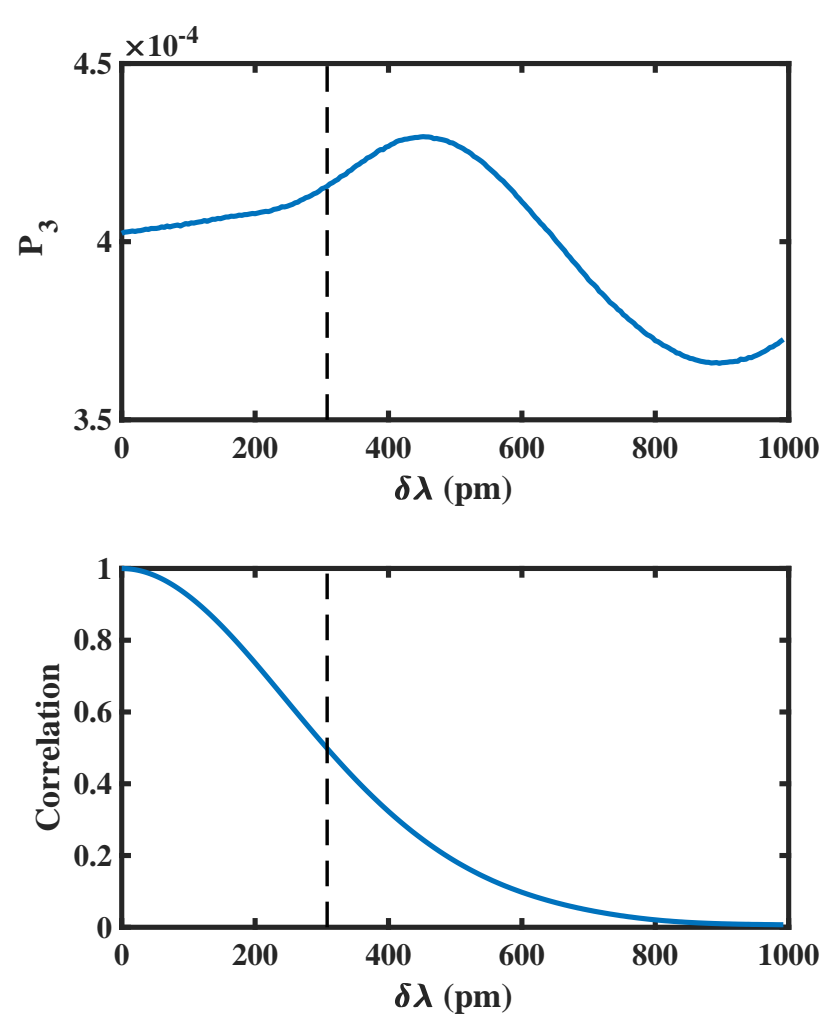

Figure 1: The Poincaré descriptor $\mathrm{P}_{k}$ (top) and speckle correlation (bottom) variation with wavelength, where $\delta \lambda=0$ corresponds to a wavelength of $780 \mathrm{~nm}$ for simulated data. The speckle correlation limit, above which the transmission matrix method is a reliable tool for speckle-based wavelength measurement, is indicated by the vertical dashed line. $\mathrm{P}_{k}$ is monotonic over a wavelength range comparable to this speckle correlation limit, and therefore provides a reliable probe of wavelength below this limit.

$2 \times 3$ crossover) using saturated absorption spectroscopy, leading to a central wavelength of $780.244 \mathrm{~nm}$. In order to applied a controlled variation to the wavelength of the beam, we frequency-modulate the drive voltage of an acousto-optic modulator (AOM) (Crystal Tech 3110120). The light is subsequently coupled into an anglecleaved single-mode fibre (SMF) (ThorLabs P5-780PMFC-10), eliminating any spatial variations in the beam. The SMF delivers $1.5 \mathrm{~mW}$ via a physical-contact connector (ThorLabs SM1FCA) to a Spectralon-coated integrating sphere (Ocean Optics FOIS-1, diameter of $38.1 \mathrm{~mm}$, output aperture diameter of $9.5 \mathrm{~mm}$ ). The hollow sphere has a diffusely reflecting inner surface which causes the beam to disperse over time 33. After multiple reflections, light escapes through the sphere's single output aperture and propagates in free space. The resulting speckle pattern is captured by a fast camera (Mikrotron EoSens 4CXP 4 megapixel CMOS camera) and sent to a computer to be analysed. The typical camera settings are a frame rate of $250 \mathrm{fps}$, an image size of $256 \times 256$ pixels, and an exposure of $8 \mathrm{~ms}$, and the distance between sphere and camera is chosen to be $17 \mathrm{~cm}$ to ensure speckle grains are larger
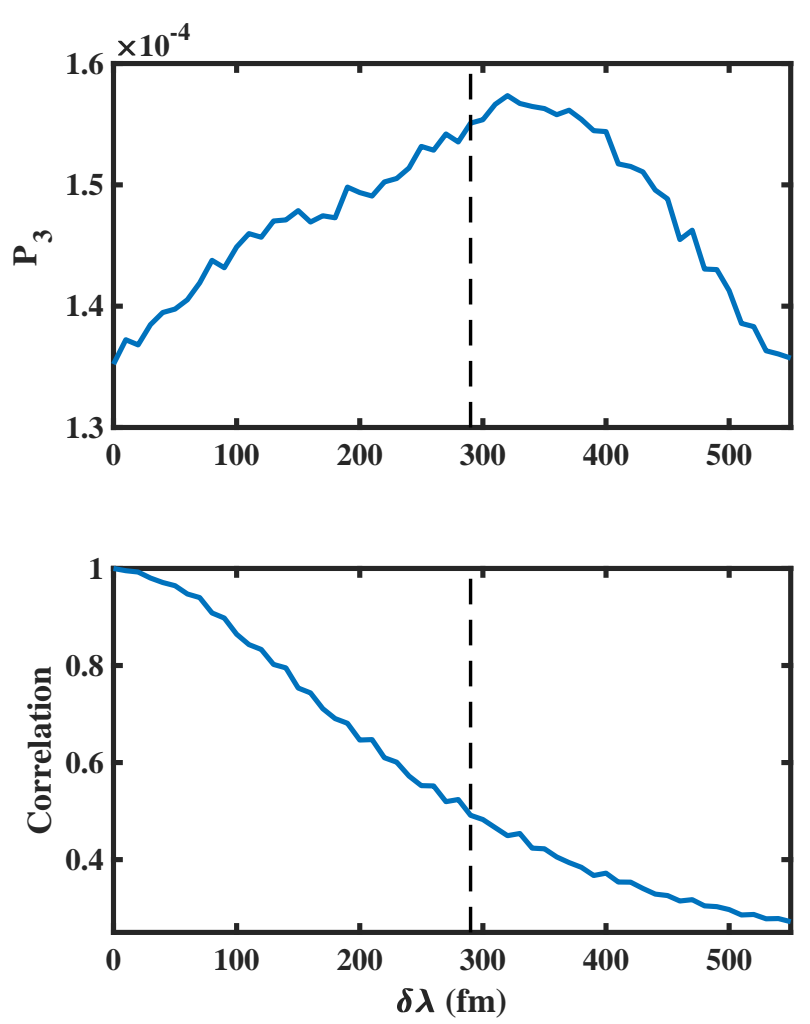

Figure 2: The Poincaré descriptor $\mathrm{P}_{k}$ (top) and speckle correlation (bottom) variation with wavelength for experimental data, where $\delta \lambda=0$ corresponds to a wavelength of $780.244 \mathrm{~nm}$. As in simulation, $\mathrm{P}_{k}$ is monotonic over a wavelength range comparable to the speckle correlation limit (vertical dashed line), which is $290 \mathrm{fm}$.

than the camera pixels. For Poincaré analysis, we use $k=3$ to match the size of a typical speckle grain from the sphere. In Figure 2 we show $P_{3}(\lambda)$ and the speckle correlation curve, verifying the finding of the simulation that $P_{k}$ is monotonically-varying over a range comparable to the speckle correlation length, which is $290 \mathrm{fm}$. To measure wavelength, training is performed by recording the speckle patterns produced during 3 periods of a sawtooth waveform which modulates the wavelength over a range of $40 \mathrm{fm}$ at $50 \mathrm{~Hz}$ (i.e. the total training duration was $60 \mathrm{~ms}$ ). From these, we extract $P_{3}(\lambda)$ for use in subsequent measurements. In Figure 3 we show the measurement of wavelength when a sinusoidal modulation is applied to the AOM with an amplitude of $10.7 \mathrm{fm}$ and a frequency of $10 \mathrm{~Hz}$. The modulation is recovered with a signal-to-noise ratio of 3.1 , i.e. a resolution of $3.3 \mathrm{fm}$.

\section{Comparison to Principal Component Analysis}

While the Poincaré descriptor method performs femtometer-resolved measurements, speckle wavemeters have previously been shown to resolve wavelength changes in the attometer-regime 20]. Such high resolution required the use of Principal Component Analysis (PCA), a mul- 


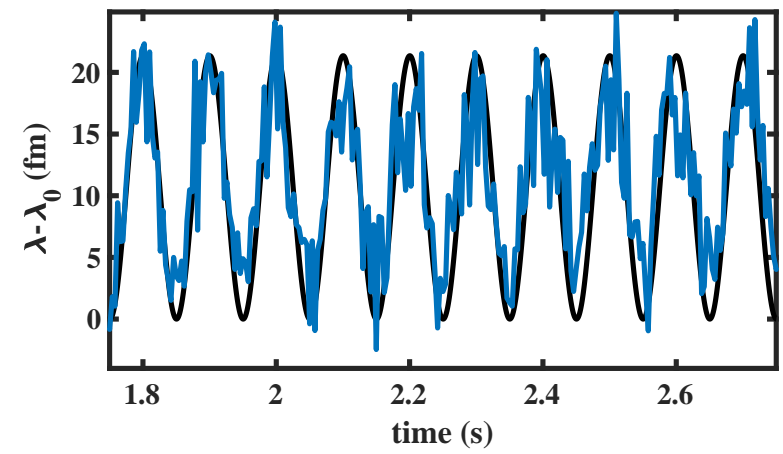

Figure 3: In experiment, the Poincaré descriptor method can measure wavelength changes on the femtometer scale. A sinusoidal wavelength modulation with amplitude $10.7 \mathrm{fm}$ (black) is applied using an acousto-optic modulator, and the resulting changes in the speckle pattern are measured using the Poincaré descriptor $P_{3}$ (blue), achieving a signal-to-noise ratio of 3.1 .

tivariate data analysis tool designed to identify a measurement basis from which maximal information can be extracted. For the system reported here, the wavelength is recovered with a resolution of $0.2 \mathrm{fm}$.

PCA re-orients a high-dimensional dataset (i.e. the images of the speckle patterns produced as the wavelength varies) into an optimal basis from which the largest variations in the data are identified from comparatively few parameters (the Principal Components or PCs). As with Poincaré analysis, in order to extract unknown wavelengths, a training set of speckle patterns at known wavelengths must be collected. Each individual speckle pattern in the training set is flattened into a 1D array and independently normalised and the set is used to build up a matrix $A$, with each of the columns corresponding to an individual speckle pattern of a particular wavelength. The PCs of the training set are the eigenvectors $v$ forming the eigenbasis of the covariance matrix $M=A^{T} A$, where $A^{T}$ is the transpose of $A$, and these eigenvectors are sorted by decreasing magnitude of their corresponding eigenvalue. The calibration matrix $T_{c a l}$ relating PCs to wavelength is given by $T_{c a l}=M v$. As the biggest variations in the training data are contained in the first few PCs, $T_{\text {cal }}$ can be down-sampled to a dimensionality of

(the number of speckle images in the training set)

$\times$ (the desired number of PCs).

Once this training procedure has been carried out, unknown wavelengths can be deduced by projecting their speckle patterns into the principal component space of the training set,

$$
\lambda=I_{x, y}(\lambda) T_{\text {cal }} .
$$

The accuracy and range of the method depends on the density and number of images in the training set [14, but is improved at the cost of computation speed.

To quantify the comparative speed of PCA and Poincaré analysis, we perform a wavelength measurement using a
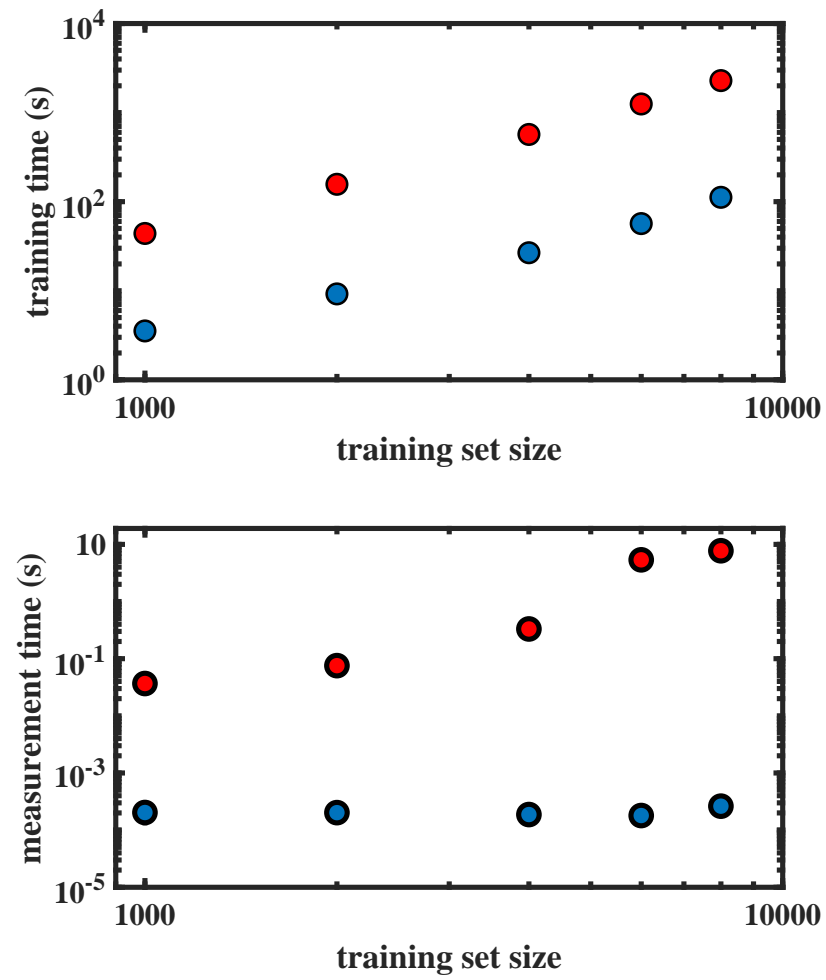

Figure 4: Comparative measurement of the speed of PCA and of the Poincaré descriptor method. (a) The time required to train PCA (red) and Poincaré analysis with $k=1$ (blue) both exhibit powerlaw growth with the size of the training set. The training duration in Poincaré analysis is reduced by one order of magnitude compared with PCA. (b) After training has been performed, the time taken to perform an individual measurement is independent of training set size for Poincaré analysis, while the time for an individual wavelength measurement with PCA is at least two orders of magnitude longer and increases with training set size.

common set of speckle images using both approaches. For PCA this included solely calculating the PCs and for the Poincaré analysis this included finding $P_{k}$ for all frames. We performed Poincaré analysis for a range of values from $k=1$ to $k=200$, and found no dependence of the calculation time on $k$. We present data here for $k=1$. The analysis was performed on an early 2014 model MacBook Air (4Gb RAM, $1.4 \mathrm{GHz}$ processor), which has a comparable processor speed to available micro-controllers such as Raspberry Pi. We compare the speed for both of the necessary experiment phases for recording wavelength in a speckle wavemeter: the training phase and the measurement phase.

The time required for a training phase $t_{t}$ is shown in Figure 4(a), for training set size $s$ varying from 1000 images to 8000 images. The run time of both PCA and Poincaré analysis grow according to a power-law relationship, with

$$
\begin{aligned}
t_{t}^{\mathrm{PCA}} & =(9 \pm 3) \times 10^{-5} \times s^{(1.9 \pm 0.2)}, \\
t_{t}^{P_{1}} & =(6 \pm 3) \times 10^{-5} \times s^{(1.6 \pm 0.1)},
\end{aligned}
$$


where $t_{t}$ is the average time taken over three runs. The training duration in PCA is consistently larger by more than one order of magnitude.

The time taken to perform a wavelength measurement of a single speckle pattern using each technique was also found using the same MacBook Air. In the Poincaré analysis, the act of performing a measurement involves taking a particular speckle pattern, normalising it, flattening it and then finding the corresponding $P_{k}$ values. In PCA, the act of performing a measurement involves taking a particular speckle pattern and performing the projection into the $\mathrm{PC}$ space of the training set using equation 3 . As shown in Figure 4(b), for the Poincaré analysis the speed of measurement does not vary with training set size. However, for PCA the time to perform a measurement is significantly longer, and grows with training set size. Even for a modest training set comprising 1000 unique wavelengths, the measurement of an individual unknown wavelength takes 37(1) ms with PCA, more than two orders of magnitude longer than the 230(40) ps-duration measurement with Poincaré analysis. For this particular laptop, these correspond to maximum measurement rates of $27 \mathrm{~Hz}$ for PCA and $4.3 \mathrm{kHz}$ for Poincaré analysis.

In addition to the speed difference between PCA and Poincaré analysis achievable in a microprocessor, Poincaré analysis offers another potential advantage: the ability to carry out the analysis in an integrated circuit, which could be implemented on-board certain high-end cameras to remove the need for image transfer to a computer. To our knowledge, efficient computation of PCA in an integrated circuit has not been demonstrated without the use of microprocessors. However, the Poincaré descriptor $P_{k}$ is arithmetically simpler, making it possible to perform using circuitry. $P_{k}$ can be rearranged [34] as

$$
P_{k}=\sqrt{\frac{\operatorname{var}\left(\tilde{I}_{x}\right)+\operatorname{var}\left(\tilde{I}_{x+k}\right)-2 \operatorname{cov}\left(\tilde{I}_{x}, \tilde{I}_{x+k}\right)}{2}},
$$

where $\tilde{I}_{x}=\sum_{y} I_{x, y}$ and var and cov are the variance and covariance respectively, both of which have been demonstrated to be calculable with FPGA (Field Programmable Gate Array) boards [35, 36].

\section{Conclusion}

Poincaré analysis of the laser speckle produced by an integrating sphere offers a method of measuring wavelength at femtometer-level resolution over a range of $400 \mathrm{pm}$. The method is complimentary to the widespread transmission matrix method, which provides accurate wavelength measurement over a wider range but with a resolution comparable to the range of the Poincaré analysis. Additionally, the method computes significantly faster on a microprocessor than the state-of-the-art Principal Component Analysis, opening up the possibility for high-speed $(>4 \mathrm{kHz})$ real-time measurements of wavelength from speckle which will allow faster feedback locking to the current of diode lasers. A further route to the miniaturisation of speckle wavemeters is the possibility of removing the need for a computer or microcontroller to analyse images altogether, and perform the analysis with FPGA boards. In future, the method may be extended to measure not only the wavelength of a monochromatic laser, but the spectrum of broadband light [18, or other parameters of the laser beam such as polarization 9] or transverse mode composition [10, 13.

\section{Acknowledgements}

We thank Donatella Cassettari, Yoshihiko Arita, Morgan Facchin, Paloma Rodríguez-Sevilla and Philip Wijesinghe for technical assistance and useful discussions, and acknowledge funding from the Leverhulme Trust Research Project Grant RPG-2017-197. Research data supporting this publication can be accessed at https://doi.org/10. 17630/4c8eb422-8204-4a3f-ab84-3630070dff2d

\section{References}

[1] H. Rubinsztein-Dunlop, A. Forbes, M. V. Berry, M. R. Dennis, D. L. Andrews, M. Mansuripur, C. Denz, C. Alpmann, P. Banzer, T. Bauer, et al., Roadmap on structured light, Journal of Optics 19 (2016) 013001.

[2] J. Billy, V. Josse, Z. Zuo, A. Bernard, B. Hambrecht, P. Lugan, D. Clément, L. Sanchez-Palencia, P. Bouyer, A. Aspect, Direct observation of Anderson localization of matter waves in a controlled disorder, Nature 453 (2008) 891.

[3] J.-y. Choi, S. Hild, J. Zeiher, P. Schauß, A. Rubio-Abadal, T. Yefsah, V. Khemani, D. A. Huse, I. Bloch, C. Gross, Exploring the many-body localization transition in two dimensions, Science 352 (2016) 1547-1552.

[4] S. J. Thomson, L. S. Walker, T. L. Harte, G. D. Bruce, Measuring the Edwards-Anderson order parameter of the Bose glass: A quantum gas microscope approach, Physical Review A 94 (2016) 051601.

[5] J. C. Dainty, Laser speckle and related phenomena, Springer, 2013.

[6] G. H. Kaufmann, Advances in speckle metrology and related techniques, John Wiley \& Sons, 2011.

[7] D. A. Boas, A. K. Dunn, Laser speckle contrast imaging in biomedical optics, Journal of Biomedical Optics 15 (2010) 011109.

[8] D. Briers, D. D. Duncan, E. R. Hirst, S. J. Kirkpatrick, M. Larsson, W. Steenbergen, T. Stromberg, O. B. Thompson, Laser speckle contrast imaging: theoretical and practical limitations, Journal of Biomedical Optics 18 (2013) 066018.

[9] T. W. Kohlgraf-Owens, A. Dogariu, Transmission matrices of random media: means for spectral polarimetric measurements, Optics Letters 35 (2010) 2236-2238.

[10] M. Mazilu, A. Mourka, T. Vettenburg, E. M. Wright, K. Dholakia, Simultaneous determination of the constituent azimuthal and radial mode indices for light fields possessing orbital angular momentum, Applied Physics Letters 100 (2012) 231115.

[11] B. Redding, H. Cao, Using a multimode fiber as a highresolution, low-loss spectrometer, Optics Letters 37 (2012) 3384-3386.

[12] B. Redding, S. F. Liew, R. Sarma, H. Cao, Compact spectrometer based on a disordered photonic chip, Nature Photonics 7 (2013) 746 .

[13] A. Mourka, M. Mazilu, E. Wright, K. Dholakia, Modal characterization using Principal Component Analysis: application to 
Bessel, higher-order Gaussian beams and their superposition, Scientific Reports 3 (2013) 1422.

[14] M. Mazilu, T. Vettenburg, A. Di Falco, K. Dholakia, Random super-prism wavelength meter, Optics Letters 39 (2014) 96-99.

[15] B. Redding, M. Alam, M. Seifert, H. Cao, High-resolution and broadband all-fiber spectrometers, Optica 1 (2014) 175-180.

[16] M. Chakrabarti, M. L. Jakobsen, S. G. Hanson, Speckle-based spectrometer, Optics Letters 40 (2015) 3264-3267.

[17] N. K. Metzger, R. Spesyvtsev, G. D. Bruce, B. Miller, G. T. Maker, G. Malcolm, M. Mazilu, K. Dholakia, Harnessing speckle for a sub-femtometre resolved broadband wavemeter and laser stabilization, Nature Communications 8 (2017) 15610.

[18] H. Cao, Perspective on speckle spectrometers, Journal of Optics 19 (2017) 060402.

[19] I. Alexeev, J. Wu, M. Karg, Z. Zalevsky, M. Schmidt, Determination of laser beam focus position based on secondary speckles pattern analysis, Applied Optics 56 (2017) 7413-7418.

[20] G. D. Bruce, L. O'Donnell, M. Chen, K. Dholakia, Overcoming the speckle correlation limit to achieve a fiber wavemeter with attometer resolution, Opt. Lett. 44 (2019) 1367-1370.

[21] Z. Meng, J. Li, C. Yin, T. Zhang, Z. Yu, M. Tang, W. Tong, $\mathrm{K}$. Xu, Multimode fiber spectrometer with scalable bandwidth using space-division multiplexing, AIP Advances 9 (2019) 015004.

[22] G. D. Bruce, L. O’Donnell, M. Chen, M. Facchin, K. Dholakia, Femtometer-resolved simultaneous measurement of multiple laser wavelengths in a speckle wavemeter, arXiv: 1910.13411 (2019).

[23] R. K. Gupta, G. D. Bruce, S. J. Powis, K. Dholakia, Deep learning enabled laser speckle wavemeter with a high dynamic range, arXiv: 1910.10702 (2019).

[24] L. Couturier, I. Nosske, F. Hu, C. Tan, C. Qiao, Y. Jiang, P. Chen, M. Weidemüller, Laser frequency stabilization using a commercial wavelength meter, Review of Scientific Instruments 89 (2018) 043103.

[25] S. G. Hanson, M. L. Jakobsen, M. Chakrabarti, The dynamic speckle-based wavemeter, in: Speckle 2018: VII International Conference on Speckle Metrology, volume 10834, International Society for Optics and Photonics, 2018, p. 108342D.

[26] A. Majumdar, S. J. Kirkpatrick, Spatial Poincaré plots as descriptors of speckle pattern second-order statistics, Journal of Biomedical Photonics \& Engineering 3 (2017) 030501.

[27] A. Majumdar, S. J. Kirkpatrick, Poincaré descriptors of long and short-range correlations in the spatial and temporal fluctuations of coherently scattered light, in: Dynamics and Fluctuations in Biomedical Photonics XV, volume 10493, SPIE, 2018, p. 1049305.

[28] J. von Neumann, R. H. Kent, H. R. Bellinson, B. I. Hart, The mean square successive difference, The Annals of Mathematical Statistics 12 (1941) 153-162.

[29] P. K. Stein, M. S. Bosner, R. E. Kleiger, B. M. Conger, Heart rate variability: a measure of cardiac autonomic tone, American Heart Journal 127 (1994) 1376-1381.

[30] M. Brennan, M. Palaniswami, P. Kamen, Do existing measures of Poincaré plot geometry reflect nonlinear features of heart rate variability?, IEEE Transactions on Biomedical Engineering 48 (2001) 1342-1347.

[31] N. K. Metzger, E. M. Wright, K. Dholakia, Theory and simulation of the bistable behaviour of optically bound particles in the mie size regime, New Journal of Physics 8 (2006) 139-139.

[32] A. Parretta, G. Calabrese, About the definition of "multiplier" of an integrating sphere, Int. J. Opt. \& Appl. 3 (2013) 119-124.

[33] G. D. Boreman, Y. Sun, A. B. James, Generation of laser speckle with an integrating sphere, Optical Engineering 29 (1990) 339-343.

[34] L. A. Asimow, M. M. Maxwell, Probability and statistics with applications: A problem solving text, Actex Publications, 2010.

[35] D. G. Bailey, M. J. Klaiber, Efficient hardware calculation of running statistics, in: 2013 28th International Conference on Image and Vision Computing New Zealand (IVCNZ 2013), IEEE, 2013, pp. 196-201.
[36] S. Martelli, D. Tosato, M. Cristani, V. Murino, Fast FPGAbased architecture for pedestrian detection based on covariance matrices, in: 18th IEEE International Conference on Image Processing, IEEE, 2011, pp. 389-392. 